\title{
Positive periodic solutions for a class of first-order iterative differential equations with an application to a hematopoiesis model
}

\author{
AHLÈME BOUAKKAZ
}

ABSTRACT. In this paper, a first-order iterative functional differential equation is investigated. With the help of the Schauder's fixed point theorem, we established some sufficient criteria that ensure the existence of positive periodic solutions. In addition, an application to three hematopoiesis models is also provided to corroborate the effeteness of our main findings. These last ones substantially enrich and complement some earlier works.

\section{INTRODUCTION}

Undoubtedly, iterative differential equations which are a special kind of differential equations involving time and state-dependent delays can be found in all areas of science and appear frequently in life sciences where the delays cannot be neglected and generally depend on both the time and the state variable (see [23]). For example, in models of hematopoiesis, the delay which represents the cell cycle duration depends, in fact, on both the time $t$ and the density of mature cells. In the models of insect population dynamics, the delay describes the life cycle period and depends also on the time $t$ and the population size. This dependence on the state variable is due to the competition among larvae for habitat and food during larval stage.

The literature of such kind of equations is still limited, because, despite the great efforts that have been made so far to overcome the difficulties in studying them, such as those caused by the iterative terms, a lot still remains to be done to construct a fully established theory. Very recently, some authors have sought to deal with these equations. For instance, concerning first-order iterative differential equations, the author of this article and her co-author R. Khemis [7] discussed the existence of solutions for a first order delayed differential equation with an iterative source term that modelizes the evolution of an insect population, M. Fečkan and al. [10] recently studied the maximal and minimal nondecreasing bounded solutions for a first order iterative differential equation. The situation is the same with the higher order equations as very few contributions that have been made so far (see $[4,5,6,8,13,14])$.

In the current manuscript, we focus on the following first-order iterative functional differential equation:

$$
x^{\prime}(t)=-a(t) x(t)+p(t) x^{m}(t-\tau(t)) f\left(t, x^{[1]}(t), x^{[2]}(t), \ldots, x^{[n]}(t)\right),
$$

where $x^{[i]}(t)$ are the iterates of $x(t), \tau, a, p \in \mathcal{C}\left(\mathbb{R}, \mathbb{R}^{+}\right)$are $T$-periodic functions, $f \in \mathcal{C}\left(\mathbb{R}^{n+1}, \mathbb{R}^{+}\right)$and $m \geq 0$. This class of equations arises in relation to the population dynamics of the Australian sheep blowfly Lucilia cuprina in ecology and the blood cell dynamics in biology. Our main task here is to prove the existence of positive periodic solutions of equation (1.1) which include a time varying delay $\tau(t)$ and several implicit

Received: 08.03.2021. In revised form: 19.07.2021. Accepted: 26.07.2021

2010 Mathematics Subject Classification. 34K13, 34A34, 34K30, 34L30.

Key words and phrases. Iterative functional differential equation, Hematopoiesis model, Fixed point theorem. 
delays depending on time and state of the form $\tau_{i}(t, x(t))$ that gave rise to the iterates $x^{[2]}(t), \ldots, x^{[n]}(t)$.

The remainder of the current article is planned as follows. In the next Section, we will present and prove a useful inequality needed to achieve the desired results. The third section is the core of the paper, where we will use Schauder's fixed point theorem to discuss the existence of positive periodic solutions of equation (1.1). The fourth section will be devoted to present some examples of models of hematopoiesis to validate our obtained results. We will end the paper with a conclusion.

\section{PRELIMINARIES}

For $\alpha, \beta \geq 0$ and $T>0$, we consider the following closed convex and bounded subset

$$
P_{T}(\alpha, \beta)=\left\{x \in P_{T}, 0 \leq x \leq \alpha,\left|x\left(t_{2}\right)-x\left(t_{1}\right)\right| \leq \beta\left|t_{2}-t_{1}\right|, \forall t_{1}, t_{2} \in \mathbb{R}\right\},
$$

of the following Banach space

$$
P_{T}=\{x \in \mathcal{C}(\mathbb{R}, \mathbb{R}), x(t+T)=x(t)\},
$$

with the norm

$$
\|x\|=\sup _{t \in[0, T]}|x(t)| .
$$

$P_{T}(\alpha, \beta)$ is a compact subset of $P_{T}$, this actually results from applying the Arzelá-Ascoli theorem.

Now, we will prove the next lemma which has an important role in establishing our main results.

Lemma 2.1. If $x, y \in P_{T}(\alpha, \beta)$, then

$$
\left\|x^{r}-y^{r}\right\| \leq r \alpha^{r-1}\|x-y\| .
$$

Proof. We will prove this inequality by induction. So, the proof will now proceed in two steps:

The basis step: For $r=1$, we have

$$
\|x-y\| \leq\|x-y\|,
$$

then, the inequality holds for $r=1$.

The inductive step: Now, we assume that the inequality holds for a given $r=k-1$, and we want to show that it also holds for $r=k$. Suppose that

$$
\left\|x^{k-1}-y^{k-1}\right\| \leq(k-1) \alpha^{k-2}\|x-y\| .
$$

Then

$$
\begin{aligned}
\left\|x^{k}-y^{k}\right\| & =\left\|x^{k}-x^{k-1} y+x^{k-1} y-y^{k}\right\| \\
& \leq\left\|x^{k-1}\right\|\|x-y\|+\|y\|\left\|x^{k-1}-y^{k-1}\right\| .
\end{aligned}
$$

Using (2.3), we obtain

$$
\begin{aligned}
\left\|x^{k}-y^{k}\right\| & \leq \alpha^{k-1}\|x-y\|+\alpha(k-1) \alpha^{k-2}\|x-y\| \\
& =k \alpha^{k-1}\|x-y\| .
\end{aligned}
$$




\section{EXISTENCE OF PERIODIC SOLUTIONS}

If $a(t) \neq 0$ and $G(t, \sigma)=\frac{\exp \left(\int_{t}^{\sigma} a(u) d u\right)}{\exp \left(\int_{0}^{T} a(u) d u\right)-1}, x \in P_{T}(\alpha, \beta)$ is a solution of (1.1) if and only if $x$ is a solution of the following integral equation:

$$
x(t)=\int_{t}^{t+T} G(t, \sigma)\left[p(\sigma) x^{m}(\sigma-\tau(\sigma)) f\left(\sigma, x^{[1]}(\sigma), x^{[2]}(\sigma), \ldots, x^{[n]}(\sigma)\right)\right] d \sigma,
$$

where the kernel is bounded as follows:

$$
0<A=\frac{\exp \left(-\int_{0}^{T} a(u) d u\right)}{\exp \left(\int_{0}^{T} a(u) d u\right)-1} \leq G(t, \sigma) \leq \frac{\exp \left(\int_{0}^{T} a(u) d u\right)}{\exp \left(\int_{0}^{T} a(u) d u\right)-1}=B .
$$

According to [[25], Lemma 4] and the mean value theorem, we have

$$
\int_{t_{1}}^{t_{1}+T}\left|G\left(t_{2}, \sigma\right)-G\left(t_{1}, \sigma\right)\right| d \sigma \leq B T\|a\|\left|t_{2}-t_{1}\right|, \forall t_{1}, t_{2} \in \mathbb{R} .
$$

Furthermore, we assume that function $f\left(t, \xi_{1}, \ldots, \xi_{n}\right)$ is fulfilling the following conditions:

$$
f\left(t+T, \xi_{1}, \xi_{2}, \ldots, \xi_{n}\right)=f\left(t, \xi_{1}, \xi_{2}, \ldots, \xi_{n}\right),
$$

and there exist $n$ positive constants $\ell_{1}, \ell_{2}, \ldots, \ell_{n}$ such that

$$
\left|f\left(t, \xi_{1}, \ldots, \xi_{n}\right)-f\left(t, \xi_{1}^{*}, \ldots, \xi_{n}^{*}\right)\right| \leq \sum_{i=1}^{n} \ell_{i}\left|\xi_{i}-\xi_{i}^{*}\right|
$$

Moreover, for $p \in P_{T}\left(\alpha_{p}, \beta_{p}\right)$, we consider the following two assumptions:

$$
T B \alpha_{p} \alpha^{m+1} \ell_{0} \sum_{i=1}^{n} \ell_{i} \sum_{j=0}^{j=i-1} \beta^{j} \leq \alpha
$$

and

$$
(2+T\|a\|) B \alpha_{p} \alpha^{m}\left(\ell_{0}+\alpha \sum_{i=1}^{n} \ell_{i} \sum_{j=0}^{j=i-1} \beta^{j}\right) \leq \beta
$$

To apply Schauder's fixed point theorem, we will need to construct a compact operator. Thus, from (3.4), we set the operator $\mathcal{H}: P_{T}(\alpha, \beta) \rightarrow P_{T}$ as follows:

$$
(\mathcal{H} \varphi)(t)=\int_{t}^{t+T} G(t, \sigma)\left[p(\sigma) \varphi^{m}(\sigma-\tau(\sigma)) f\left(\sigma, \varphi^{[1]}(\sigma), \varphi^{[2]}(\sigma), \ldots, \varphi^{[n]}(\sigma)\right)\right] d \sigma .
$$

Theorem 3.1. Let $a(t) \neq 0$. If $p \in P_{T}\left(\alpha_{p}, \beta_{p}\right)$ and (3.7) - (3.10) are satisfied, then equation (1.1) has a positive periodic solution in $P_{T}(\alpha, \beta)$. 
Proof. If $\varphi, \phi \in P_{T}(\alpha, \beta)$ then

$$
\begin{aligned}
|(\mathcal{H} \varphi)(t)-(\mathcal{H} \phi)(t)| & =\int_{t}^{t+T} G(t, \sigma) p(\sigma) \mid \varphi^{m}(\sigma-\tau(\sigma)) f\left(\sigma, \varphi^{[1]}(\sigma), \ldots, \varphi^{[n]}(\sigma)\right) \\
& -\phi^{m}(\sigma-\tau(\sigma)) f\left(\sigma, \varphi^{[1]}(\sigma), \ldots, \varphi^{[n]}(\sigma)\right) \\
& +\phi^{m}(\sigma-\tau(\sigma)) f\left(\sigma, \varphi^{[1]}(\sigma), \ldots, \varphi^{[n]}(\sigma)\right) \\
& -\phi^{m}(\sigma-\tau(\sigma)) f\left(\sigma, \phi^{[1]}(\sigma), \ldots, \phi^{[n]}(\sigma)\right) \mid d \sigma \\
& \leq \int_{t}^{t+T} G(t, \sigma) p(\sigma)\left|\varphi^{m}(\sigma-\tau(\sigma))-\phi^{m}(\sigma-\tau(\sigma))\right| \\
& \times\left|f\left(\sigma, \varphi^{[1]}(\sigma), \ldots, \varphi^{[n]}(\sigma)\right)\right| d s \\
& +\int_{t}^{t+T} G(t, \sigma) p(\sigma)\left|\phi^{m}(\sigma-\tau(\sigma))\right| f\left(\sigma, \varphi^{[1]}(\sigma), \ldots, \varphi^{[n]}(\sigma)\right) \\
& -f\left(\sigma, \phi^{[1]}(\sigma), \ldots, \phi^{[n]}(\sigma)\right) \mid d \sigma .
\end{aligned}
$$

It follow from (3.8) that

$$
\begin{aligned}
\left|f\left(\sigma, \varphi^{[1]}(\sigma), \varphi^{[2]}(\sigma), \ldots, \varphi^{[n]}(\sigma)\right)\right| & =\mid f\left(\sigma, \varphi^{[1]}(\sigma), \varphi^{[2]}(\sigma), \ldots, \varphi^{[n]}(\sigma)\right) \\
& -f(\sigma, 0, \ldots, 0)+f(\sigma, 0, \ldots, 0) \mid \\
& \leq\left|f\left(\sigma, \varphi^{[1]}(\sigma), \varphi^{[2]}(\sigma), \ldots, \varphi^{[n]}(\sigma)\right)-f(\sigma, 0, \ldots, 0)\right| \\
& +|f(\sigma, 0, \ldots, 0)| \\
& =\ell_{0}+\alpha \sum_{i=1}^{n} \ell_{i} \sum_{j=0}^{j=i-1} \beta^{j},
\end{aligned}
$$

where $\ell_{0}=\sup _{\sigma \in[0, T]}|f(\sigma, 0, \ldots, 0)|$.

Using (2.2), (3.5), [[26], Lemma 2.1] and (3.12) we obtain

$$
|(\mathcal{H} \varphi)(t)-(\mathcal{H} \phi)(t)| \leq \beta T \alpha_{p}\left(\alpha\left(m \alpha^{m-1}+1\right)\left(\sum_{i=1}^{n} \ell_{i} \sum_{j=0}^{j=i-1} \beta^{j}\right)+m \alpha^{m-1} \ell_{0}\right)\|\varphi-\phi\| .
$$

\section{Consequently, $\mathcal{H}$ is a continuous operator.}

On the other hand, if $\varphi \in P_{T}(\alpha, \beta)$ we have

$$
|\mathcal{H}(\varphi(t))|=\int_{t}^{t+T} G(t, \sigma)\left[p(\sigma) \varphi^{m}(\sigma-\tau(\sigma)) f\left(\sigma, \varphi^{[1]}(\sigma), \ldots, \varphi^{[n]}(\sigma)\right)\right] d \sigma,
$$

and from (3.5) and (3.12), we arrive at

$$
|\mathcal{H}(\varphi(t))| \leq T B \alpha_{p} \alpha^{m+1} \ell_{0} \sum_{i=1}^{n} \ell_{i} \sum_{j=0}^{j=i-1} \beta^{j} .
$$

Using now (3.9), we deduce that

$$
\|\mathcal{H} \varphi\|=\sup _{t \in[0, T]}|\mathcal{H}(\varphi(t))| \leq \alpha
$$


Now, let $t_{1}, t_{2} \in \mathbb{R}$ (with $t_{1}<t_{2}$ ), so

$$
\begin{aligned}
\left|\mathcal{H}\left(\varphi\left(t_{1}\right)\right)-\mathcal{H}\left(\varphi\left(t_{2}\right)\right)\right| & \leq \int_{t_{2}}^{t_{1}} G\left(t_{2}, \sigma\right)\left[p(\sigma) \varphi^{m}(\sigma-\tau(\sigma)) f\left(\sigma, \varphi^{[1]}(\sigma), \ldots, \varphi^{[n]}(\sigma)\right)\right] d \sigma \\
& +\int_{t_{1}+T}^{t_{2}+T} G\left(t_{2}, \sigma\right)\left[p(\sigma) \varphi^{m}(\sigma-\tau(\sigma)) f\left(\sigma, \varphi^{[1]}(\sigma), \ldots, \varphi^{[n]}(\sigma)\right)\right] d \sigma \\
& +\int_{t_{1}}^{t_{1}+T}\left|G\left(t_{2}, \sigma\right)-G\left(t_{1}, \sigma\right)\right| p(\sigma) \varphi^{m}(\sigma-\tau(\sigma)) \\
& \times f\left(\sigma, \varphi^{[1]}(\sigma), \ldots, \varphi^{[n]}(\sigma)\right) d \sigma .
\end{aligned}
$$

Thanks again to (3.5) and (3.12) and using (3.6), we get

$$
\begin{aligned}
\left|\mathcal{H}\left(\varphi\left(t_{1}\right)\right)-\mathcal{H}\left(\varphi\left(t_{2}\right)\right)\right| & \leq 2 B \alpha_{p} \alpha^{m}\left(\ell_{0}+\alpha \sum_{i=1}^{n} \ell_{i} \sum_{j=0}^{j=i-1} \beta^{j}\right)\left|t_{2}-t_{1}\right| \\
& +B T\|a\| \alpha_{p} \alpha^{m}\left(\ell_{0}+\alpha \sum_{i=1}^{n} \ell_{i} \sum_{j=0}^{j=i-1} \beta^{j}\right)\left|t_{2}-t_{1}\right| \\
& =(2+T\|a\|) B \alpha_{p} \alpha^{m}\left(\ell_{0}+\alpha \sum_{i=1}^{n} \ell_{i} \sum_{j=0}^{j=i-1} \beta^{j}\right)\left|t_{2}-t_{1}\right| .
\end{aligned}
$$

By virtue of [[25], Lemma 4] and (3.10), we obtain

$$
\left|\mathcal{H}\left(\varphi\left(t_{1}\right)\right)-\mathcal{H}\left(\varphi\left(t_{2}\right)\right)\right| \leq \beta\left|t_{2}-t_{1}\right|, \forall t_{1}-t_{2} \in \mathbb{R} .
$$

Finally, from (3.13) and (3.14), we conclude that

$$
\mathcal{H}\left(P_{T}(\alpha, \beta)\right) \subset P_{T}(\alpha, \beta) .
$$

This last inclusion implies that the continuous operator $\mathcal{H}$ maps the bounded compact and convex subset $P_{T}(\alpha, \beta)$ of the Banach space $P_{T}$ into itself. Therefore, Schauder's fixed point theorem ensures that the compact operator $\mathcal{H}$ has at least one fixed point in $P_{T}(\alpha, \beta)$. Consequenly, equation (1.1) admits a positive periodic solution, residing in $P_{T}(\alpha, \beta)$.

\section{Applications to Periodic Iterative Hematopoiesis Models}

For illustrating some features of our main results, we apply the previous criteria to obtain some existence results for three Hematopoiesis Models.

Example 4.1. Consider the following iterative hematopoiesis model with periodic coefficients:

$$
x^{\prime}(t)=-a(t) x(t)+\sum_{i=1}^{n} \frac{p(t)}{1+x^{[i]}(t)} .
$$

This model which describes the production of blood cells can be considered as a particular case of $(1.1)$, where $m=0, p_{1}(t)=\ldots=p_{n}(t)=p(t)$ and $f\left(t, x^{[1]}(t), \ldots, x^{[n]}(t)\right)=$ $\sum_{i=1}^{n} \frac{1}{1+x^{[i]}(t)}$. 
Here, $x(t)$ denotes the density of mature cells in blood circulation at time $t, a(t)$ is the decay rate, $p(t)$ is the production rate, $\sum_{i=1}^{n} \frac{p(t)}{1+x^{[i]}(t)}$ is the flux of the cells into the circulation from the stem cell compartment and the iterates $x^{[i]}(t)$ result from delays of the form $\tau_{i}(t, x(t))$ which represent the times required to produce mature cells.

The Banach space, the closed, bounded and convex subset and the Green's kernel are the same as in the previous sections. While the operator $\mathcal{H}: P_{T}(\alpha, \beta) \longrightarrow P_{T}$ is given by:

$$
(\mathcal{H} \varphi)(t)=\sum_{i=1}^{n} \int_{t}^{t+T} G(t, \sigma) \frac{p(\sigma)}{1+x^{[i]}(\sigma)} d \sigma .
$$

$\mathcal{H}$ is continuous. Indeed, it's not hard to prove that

$$
|(\mathcal{H} \varphi)(t)-(\mathcal{H} \phi)(t)| \leq n \alpha_{p} T B \sum_{i=1}^{n} \sum_{j=0}^{i-1} \beta^{j}\|\varphi-\phi\| .
$$

Conditions $(3.9),(3.10)$ of theorem 3.1 become, respectively:

$$
n \alpha_{p} T B \leq \alpha,
$$

and

$$
(2+T\|a\|) n \alpha_{p} B \leq \beta .
$$

Theorem 4.2. Let us assume that $a(t) \neq 0, p \in P_{T}\left(\alpha_{p}, \beta_{p}\right)$ and that (3.7), (4.18) and (4.19) hold. Then all conditions of theorem 3.1 are fulfilled and, therefore (4.16) admits a positive periodic solution in $P_{T}(\alpha, \beta)$.

Remark 4.1. Notice that, the Lipschitz condition was dropped without any further assumptions on $f$ and, despite this, (4.16) has a positive periodic solution.

Remark 4.2. (4.16) has been studied by numerous authors in the recent decades such as Alzabut et al. [1] who investigated it with one constant delay and Liu et al. [16] who studied this equation but with multiple time-varying delays to name a few.

Example 4.2. We consider the following iterative Lasota-Wazewska type differential equation:

$$
x^{\prime}(t)=-\frac{1}{5}\left(\cos ^{4} \frac{2 \pi}{25} t\right) x(t)+\frac{1}{10}\left(\cos ^{2} \frac{2 \pi}{25} t\right) e^{-\frac{1}{8} x^{[2]}(t)},
$$

in the set $P_{T}(\alpha, \beta)=P_{25}\left(\frac{\pi}{3}, \frac{\pi}{2}\right)$. Equation (4.20) describes the survival of red blood cells in the blood of an animal where $x(t)$ is the number of red blood cells at time $t$, $\frac{1}{5}\left(\cos ^{4} \frac{2 \pi}{25} t\right)$ is the death rate of red blood cells and $\frac{1}{10}\left(\cos ^{2} \frac{2 \pi}{25} t\right)$ is related to the production of red blood cells per unit time.

By virtue of the following inequality

$$
\left|e^{-x}-e^{-y}\right| \leq|x-y|, \forall x, y \in[0, \infty[
$$

$f$ is a Lipschitz function and

$$
|f(t, x)-f(t, y)| \leq \frac{1}{8}|x-y|, \forall x, y \in P_{25}\left(\frac{\pi}{3}, \frac{\pi}{2}\right) .
$$

We have

$$
a(t)=\frac{1}{5}\left(\cos ^{4} \frac{2 \pi}{25} t\right), p(t)=\frac{1}{10}\left(\cos ^{2} \frac{2 \pi}{25} t\right), B \simeq 1.1811,\|a\|=\frac{1}{5}, m=0, \alpha_{p}=\frac{1}{10},
$$




$$
\ell_{0}=1, \ell_{2}=\frac{1}{8}, T B \alpha_{p} \alpha^{m+1} \ell_{0} \sum_{i=1}^{n} \ell_{i} \sum_{j=0}^{j=i-1} \beta^{j} \simeq 0.99365 \leq \alpha=\frac{\pi}{3} \simeq 1.0472,
$$

and

$$
(2+T\|a\|) B \alpha_{p} \alpha^{m}\left(\ell_{0}+\alpha \sum_{i=1}^{n} \ell_{i} \sum_{j=0}^{j=i-1} \beta^{j}\right) \simeq 1.1050 \leq \beta=\frac{\pi}{2} \simeq 1.5708 .
$$

Consequently, all assumptions of theorem 3.1 are satisfied and equation (4.20) has at least one solution in $P_{25}\left(\frac{\pi}{3}, \frac{\pi}{2}\right)$.

Remark 4.3. We chose a period of 25 days since the period can vary from a few weeks up to a few months especially in the case of periodic haematological disorders which have long periods ranging from 20 to 60 days.

Remark 4.4. The above equation has the same form as the equation in [12] which is with delays depending only on time.

Example 4.3. We consider the following iterative differential equation

$$
x^{\prime}(t)=-\frac{1}{9}\left(\sin ^{4} \frac{2 \pi}{33} t\right) x(t)+\frac{1}{12}\left(\cos ^{2} t\right)\left(\frac{x^{[2]}}{1+x^{[2]}}+\frac{x^{[3]}}{1+x^{[3]}}\right),
$$

in the set $P_{T}(\alpha, \beta)=P_{33}\left(\frac{1}{6}, \frac{1}{4}\right)$ where $m=0, f(t, x, y)=\frac{x}{1+x}+\frac{y}{1+y}$, $a(t)=\frac{1}{9}\left(\sin ^{4} \frac{2 \pi}{33} t\right)$ and $p(t)=\frac{1}{12}\left(\cos ^{2} t\right)$.

For $x_{1}, y_{1}, x_{2}, y_{2}$ in $P_{33}\left(\frac{1}{6}, \frac{1}{4}\right)$ we have

$$
\left|f\left(t, x_{1}, y_{1}\right)-f\left(t, x_{2}, y_{2}\right)\right|=\left|\frac{x_{1}}{1+x_{1}}-\frac{x_{2}}{1+x_{2}}+\frac{y_{1}}{1+y_{1}}-\frac{y_{2}}{1+y_{2}}\right| \leq\left|x_{1}-x_{2}\right|+\left|y_{1}-y_{2}\right|,
$$

and

$$
\begin{gathered}
\ell_{0}=0, \ell_{1}=\ell_{2}=1,\|a\|=\frac{1}{9}, B \simeq 1.3384, \alpha_{p}=\frac{1}{12}, \\
T B \alpha_{p} \alpha^{m+1} \ell_{0} \sum_{i=1}^{n} \ell_{i} \sum_{j=0}^{j=i-1} \beta^{j}=0 \leq \alpha=\frac{1}{6}, \\
(2+T\|a\|) B \alpha_{p} \alpha^{m}\left(\ell_{0}+\alpha \sum_{i=1}^{n} \ell_{i} \sum_{j=0}^{j=i-1} \beta^{j}\right)=0.23701 \leq \beta=\frac{1}{4} .
\end{gathered}
$$

It follows from theorem 3.1 that $(4.21)$ has at least one solution in $P_{33}\left(\frac{1}{6}, \frac{1}{4}\right)$.

Remark 4.5. In $[15,16,22,24]$ the authors studied a similar hematopoiesis model but with time-varying delays.

\section{CONCLUSION AND REMARKS}

This work is devoted to study a class of iterative differential equations arising in several fields of life sciences such as hematology and ecology. For transforming the existence of periodic solutions of our iterative differential equation into a fixed point problem, we chose an appropriate Banach space that ensures that the iterates terms are well-defined, 
we next converted our equation into an equivalent integral equation and with the aid of some properties of the obtained Green's kernel and the Schauder's fixed point theorem, we have been able to prove the existence of at least one positive periodic solution which need not be unique. Three examples are given to illustrate the feasibility of our findings which are a continuation of our recent papers and some known works.

The results of this manuscript are essentially new and complement some previous studies. They differ from those obtained in the papers cited below in five aspects.

(1) Several new existence results for an iterative differential equation have been presented which enrich the few existing literature on such kind of equations that have not yet been adequately studied. Our iterative differential equation is of degree $n$ whereas the equations in [2], [3], [9], [11], [13], [18] and [21] are of second degree and despite that the equations in [17], [19], [20], [25] and [26] are of degree $n$, they can be considered as a particular case of our equation. The main difficulty in studying this kind of equations is due to the iterative terms that emerge from multiple implicit delays of the form $\tau_{i}(t, x(t))$ depending not only on time, but also on the state variable $x$. Thanks to a powerful technique which combines the Green's method and Schauder's fixed point theorem together with Arzela-Ascoli theorem, we realized our objectives. In addition, aside from all the hurdles in establishing the Green's kernel and some of its properties, it is also quite difficult to make the iterates well-defined. For this we constructed an appropriate Banach space to overcome this difficulty.

(2) The considered equation often appears in the mathematical modeling of many biological phenomena such as infectious disease transmission models, Nicholson's blowflies model, models of hematopoiesis, etc. For instance, in order to illustrate some features of our main findings, we studied a more general model of hematopoiesis with complex cell cycles where the delays which represent the average cell cycle duration, depend on both the time $t$ and the current density of mature cells $x(t)$. Actually, cell cycle durations are also controlled by this latter, when the number of mature blood cells is large, some growth factors and hormones suppress the division of the hematopoietic stem cells, and in the converse case, it will be promoted. So far there are no publications about iterative hematopoiesis model which implies that the present work is of significance since it extends and improves the existing studies on hematopoiesis that are concerned only with constant or time-varying delays (see for example [1, 12, 15, 16, 22, 24]).

(3) Our results are applicable to different models, such as insect population dynamics models. So they can be regarded as a generalisation of our work [7] and references cited therein.

(4) It has also been shown that our findings can be improved by relaxing the assumptions on the source term as in example (4.1) when we relaxed conditions on $f$.

(5) Due to biological applications, the solution may represent cell number or population density. So, to obtain results that is closer to reality, it is more reasonable to show the positivity and the periodicity of solutions.

\section{REFERENCES}

[1] Alzabut, J. O.; Nieto, J. J.; Stamov, G. T. Existence and exponential stability of positive almost periodic solutions for a model of hematopoiesis. Bound. Value Probl. 2009, 127510

[2] Andrzej, P. On some iterative-differential equation I. Zeszyty Naukowe UJ. Prace Mat. 12 (1968), 53-56

[3] Berinde, V. Existence and approximation of solutions of some first order iterative differential equations. Miskolc Math. Notes 11 (2010), 13-26

[4] Bouakkaz, A.; Ardjouni, A.; Djoudi, A. Periodic solutions for a second order nonlinear functional differential equation with iterative terms by Schauder fixed point theorem. Acta Math. Univ. Comen. 87 (2018), 223-235 
[5] Bouakkaz, A.; Ardjouni, A.; Khemis, R.; Djoudi, A. Periodic solutions of a class of third-order functional differential equations with iterative source terms. Bol. Soc. Mat. Mex. 26 (2020), 443-458

[6] Bouakkaz, A.; Khemis, R. Positive periodic solutions for a class of second-order differential equations with state-dependent delays. Turk J Math. 44 (2020), 1412-1426

[7] Bouakkaz, A.; Khemis, R. Positive periodic solutions for revisited Nicholson's blowflies equation with iterative harvesting term. J. Math. Anal. Appl. 494 (2021), 124663

[8] Cheraiet, S.; Bouakkaz, A.; Khemis, R. Bounded positive solutions of an iterative three-point boundaryvalue problem with integral boundary conditions. J. Appl. Math. Comput. 65 (2021), 597-610

[9] Eder, E. The Functional Differential Equation $x^{\prime}(t)=x(x(t))$. J. Differential Equations 54 (1984), 390-400

[10] Fečkan, M.; Wang, J.; Zhao, H. Y. Maximal and minimal nondecreasing bounded solutions of iterative functional differential equations. Appl. Math. Lett. 113 (2021), 106886

[11] Ge, W.; Mo, Y. Existence of solutions to differential-iterative equation. J. Beijing Inst. Tech. 6 (1997), 192-200

[12] Huang, Z.; Gong, S.; Wang, L. Positive almost periodic solution for a class of Lasota-Wazewska model with multiple time-varying delays. Comput. Math. Appl. 61 (2011), 755-760

[13] Kaufmann, E. R. Existence and uniqueness of solutions for a second-order iterative boundary-value problem functional differential equation. Electron. J. Differ. Equ. 150 (2018), 1-6

[14] Khemis, R.; Ardjouni, A.; Bouakkaz, A.; Djoudi, A. Periodic solutions of a class of third-order differential equations with two delays depending on time and state. Comment. Math. Univ. Carolin. 60 (2019), 379-399

[15] Liu, B. New results on the positive almost periodic solutions for a model of hematopoiesis. Nonlinear Anal. Real World Appl. 17 (2014), 252-264

[16] Liu, G.; Yan, J.; Zhang, F. Existence and global attractivity of unique positive periodic solution for a model of hematopoiesis. J. Math. Anal. Appl. 334 (2007), 157-171

[17] Liu, J.; Si, J. G. Analytic solutions for a class of differential equation with delays depending on state. Appl. Math. Comput. 186 (2007), 261-270

[18] Staněk, S. On global properties of solutions of functional differential equation $x^{\prime}(t)=x(t)+x(x(t))$. Dynam. Systems Appl. 4 (1995), 263-278

[19] Si, J. G.; Li, W. R.; Cheng, S. S. Analytic solutions of an iterative functional differential equation. Comput. Math. Appl. 33 (1997), 47-51

[20] Si, J. G.; Wang, X. P. Smooth solutions of a nonhomogeneous iterative functional differential equation with variable coefficients. J. Math. Anal. Appl. 226 (1998), 377-392

[21] Wang, K. On the equation $x^{\prime}(t)=f(x(x(t)))$. Funkcial. Ekvac. 33 (1990), 405-425

[22] $\mathrm{Wu}, \mathrm{X} . ; \mathrm{Li}, \mathrm{J} . ; \mathrm{Zhou}, \mathrm{H}$. A necessary and sufficient condition for the existence of positive periodic solutions of a model of hematopoiesis. Comput. Math. Appl. 54 (2007), 840-849

[23] Yang, D.; Zhang, W. Solutions of equivariance for iterative differential equations. Appl. Math. Lett. 17 (2004), 759-765

[24] Yang, X. T. Existence and global attractivity of unique positive almost periodic solution for a model of hematopoiesis. Appl. Math. J. Chinese Univ. 25 (2010), 25-34

[25] Zhao, H. Y.; Fečkan, M. Periodic solutions for a class of differential equations with delays depending on state. Math. Commun. 23 (2018), 29-42

[26] Zhao, H. Y.; Liu, J. Periodic solutions of an iterative functional differential equation with variable coefficients. Math. Methods Appl. Sci 40 (2017), 286-292

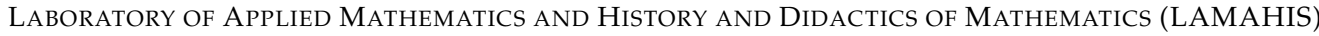
UNIVERSITY OF 20 AUGUST 1955, SKIKDA

ALGERIA

Email address: ahlemkholodedyahoo.com 